Objectives: In order to minimise diagnostic delay and improve patient care, we have implemented a questionnaire-based screening procedure in the dermatology outpatient clinic to identify patients with suspected PsA.

Methods: A questionnaire-based screening (PEST, FFbH, WHOQOL-BREF, $\mathrm{Phq9}$, GHQ-12) was used to assess $\mathrm{PsO}$ patients for PsA, depression, comorbidities, and quality of life (QOL).

Results: So far we have assessed $150 \mathrm{PsO}$ patients. $79 \%$ reported joint affection with a mean number of 4.58 involved joint regions, predominantly at the lower extremities. $34 \%$ described axial involvement. $42 \%$ had a score of $>3$ in the PEST tool, being suspicious for PsA. $4 \%$ had a clinically relevant functional impairment in daily life assessed by $\mathrm{FFbH}$ questionnaire (score <60\%). In WHOQOL-BREF $\mathrm{PsO}$ patients scored lower on the physical health as well as the psychological wellbeing scale when compared to reference values for healthy controls, while results in the social relationship and environment domains were comparable. When comparing patients with suspected PsA to PsO without arthritis the former group reached significantly lower scores in the physical health domain $(p=0.0001)$, in the psychological wellbeing $(p=0.0434)$, and environment domains $(p=0.0384)$, especially regarding items assessing body image/appearance and negative feelings for psychological wellbeing as well as physical security and mobility/transport in the environment domain. For the social domain there were no differences. In the Phq9 questionnaire patients reached a mean of 6.5 points equalling mild depressive symptoms. $16 \%$ of $\mathrm{PsO}$ patients had moderate depressive symptoms, while $9 \%$ had severe depressive symptoms. In patients with suspected PsA $44 \%$ had moderate $(26 \%)$ or severe $(18 \%)$ depression compared to $7 \%$ and $2 \%$ respectively in $\mathrm{PsO}$ patients without arthritis.

Conclusions: Screening questionnaires are valuable tools for dermatologists as well as general practitioners and help to identify PsO patients with musculoskeletal involvement pointing towards psoriatic arthritis. Thus, screening questionnaires can add to ensure timely referral of patients with inflammatory joint involvement and depression to the corresponding specialists and help to avoid a delay of treatment. Our data confirm an association of psoriasis, depressive symptoms and reduced quality of life, which was even stronger in patients with psoriatic arthritis.

Disclosure of Interest: None declared

DOI: 10.1136/annrheumdis-2018-eular.5834

\section{AB0941 PATIENT AND PHYSICIAN GLOBAL ASSESSMENTS REFLECT STRONGLY DIVERGING ATTITUDES BETWEEN PATIENTS WITH PSORIATIC ARTHRITIS AND THEIR RHEUMATOLOGISTS TO SEVERITY OF DISEASE AND TO THE RELATIVE IMPORTANCE OF DIFFERENT OUTCOME MEASURES}

O. Rintek Madsen. Center for Rheumatology and Spine Diseases and the DANBIO Registry, Copenhagen University Hospital Rigshospitalet/Gentofte, Hellerup, Denmark

Background: Assessment of disease activity is important in the evaluation and monitoring of patients with psoriatic arthritis (PsA) in clinical care and research. As there is no single 'gold standard' variable for assessment of disease activity several markers of disease activity are used, among these "global assessment" by the patient $(\mathrm{PaGl})$ and by the physician $(\mathrm{PhGl})$. The agreement and interplay between $\mathrm{PaGl}$ and $\mathrm{PhGl}$ are not well clarified in patients with PsA, however.

Objectives: The study aimed to examine associations on the group level and agreements on the individual patient level between PaGl and PhGl as scored on visual analogue scales (VAS) in the daily clinic by patients with active PsA and by their rheumatologists.

Methods: Traditional disease activity data on 76 PsA patients with active disease planned to initiate biological treatment were extracted from the Danish DANBIO registry for biological treatment in rheumatology. Data comprised $\mathrm{PaGl}, \mathrm{PhGl}$ and pain (0-100 VAS), 28 swollen joint count (SJC), 28 tender joint count (TJC), CRP, $\mathrm{HAQ}-\mathrm{DI}$ and DAS28-CRP(4 v). Analyses were performed using parametric statistics. The association between $\mathrm{PaGl}$ and $\mathrm{PhGl}$ was examined by simple linear regression analysis. The predictability of $\mathrm{PaGl}$ and $\mathrm{PhGl}$, respectively, by all other disease markers mentioned and by age and sex was examined using stepwise multiple regression analysis. Agreement between the VAS scores was expressed as the bias (mean difference between intra-individual scores) and the $95 \%$ lower and upper limits of agreement (LLoA;ULoA) according to the Bland-Altman method.

Results: Mean age was $52.2 \pm 11.1$ years and mean DAS28-CRP $4.7 \% \pm 1.1$ $59.2 \%$ of the patients were women. Mean $\mathrm{PaGl}$ was $63.7 \pm 23.2$ and mean $\mathrm{PhGl}$ $39.9 \pm 19.8(p<0.0001)$. Thus the difference between PaGl and PhGl was substantial on the group level. Differences between PaGl and PtGl were even more pronounced on the individual level, however. The average difference was 23.8 (bias) but differences on the individual level ranged from -21.9 (LLoA) to +69.5 (UloA). The corresponding results for PaGl vs. pain was 4.9 (bias), -17.1 (LLoA) and 22.0 (ULoA), and for pain vs. PhGl 18.9 (bias), -23.0 (LLoA) and 60.8 (ULoA). PaGl was significantly but weakly correlated with PhGl ( $R=0.42$ $\mathrm{p}<0.0001$ ) with a high standard error of estimation $(\mathrm{SEE})=21.2$. PaGl was independently predicted by pain (beta $=0.76, p<0.0001$ ) and HAQ-DI (beta $=0.19$ $p<0.01)$ and was not predicted by $\mathrm{PhGI}(\mathrm{p}=0.61)(\mathrm{R}=0.78$, SEE $=10.5, p<0.0001)$ $\mathrm{PhGl}$ was independently predicted by SJC (beta $=0.43, p<0.0001$ ) followed by pain (beta $=0.41, p<0.0001)$ and CRP (beta $=0.20, p<0.05)(R=0.70, S E E=14.4$ $\mathrm{p}<0.0001)$ with no significantly contribution by $\mathrm{PaGl}(\mathrm{p}=0.49)$.

Conclusions: In patients with active PsA initiating biological treatment, $\mathrm{PaGl}$ was in general scored considerably higher than PhGl. On the individual patient level, differences between $\mathrm{PaGl}$ and PhGl varied substantially. PaGl was best explained by pain, and $\mathrm{PhGl}$ by SJC. The findings reflect strongly diverging attitudes between PsA patients and their rheumatologists to severity of disease and to the relative importance of different outcome measures.

Disclosure of Interest: None declared

DOI: 10.1136/annrheumdis-2018-eular.379

\section{AB0942 CONCORDANCE BETWEEN FATIGUE, PAIN AND PATIENT GLOBAL ASSESSMENT IN INDIVIDUAL PATIENTS WITH PSORIATIC ARTHRITIS}

O. Rintek Madsen. Center for Rheumatology and Spine Diseases and the DANBIO Registry, Copenhagen University Hospital Rigshospitalet/Gentofte, Hellerup, Denmark

Background: Associations between fatigue (FTG), pain and patient global assessment ( $\mathrm{PaGl}$ ) have been examined on the group level in patients with psoriatic arthritis (PsA), but studies focusing on the concordance between these patient-reported outcome measures (PROMs) in individual patients are missing A better understanding of how tight the measures are bounded in individuals may improve our ability to deal with them in the daily clinic.

Objectives: To examine associations on the group level and concordance on the individual patient level between FTG, pain and PaGl as scored on $0-100$ visua analogue scales (VAS) in the daily clinic by patients with PSA. The influence of other clinical disease activity measures on these measures was also examined.

Methods: Data on 132 outclinic PSA patients treated with biological agents were extracted from the Danish registry for biological treatment in rheumatology (DAN $\mathrm{BIO}$ ). Data comprised VAS FTG, pain, PaGl and physician global assessment (PhGl), and HAQ-DI, swollen and tender joint counts (66/68), CRP, DAS28-CRP and age. Simple linear regression analyses were used to assess the association between FTG, pain and PaGI. Independent predictors of FTG, pain and PaG were identified using stepwise multiple regression analysis. Degrees of association were expressed by coefficients of correlation, beta-values and standard errors of estimation (SEE). Concordance between FTG, pain and PaGl on the individual patient level was examined using the Bland-Altman method yielding $95 \%$ lower and upper limits of agreement (LLoA and ULoA) and corresponding biases (mean of intra-individual differences).

Results: Mean age was $54 \pm 13$ years, mean DAS28 $3.7 \pm 1.5$ and mean PaGl 56 \pm 28 . FTG, pain and PaGl were strongly inter-associated but errors of estimation were substantial ( $r$-range $0.80-0.94, p<0.0001$, SEE-range 11.5-16.9). FTG, pain and $\mathrm{PaGl}$ were only poorly correlated with objective measures of disease activity (for example, r-range for swollen joint count $0.19-0.25, \mathrm{p}<0.05)$. FTG was independently predicted (beta, p-value) by PaGl $(0.51,<0.001)$ and pain $(0.31,<0.05)$ $\left(R^{2}=0.66, p<0.05, S E E=16.7\right)$, pain by $\mathrm{PaGl}(0.82,<0.0001)$ and $\mathrm{HAQ}-\mathrm{DI}(0.15$, $<0.005)\left(R^{2}=0.88, p<0.005\right.$, SEE $\left.=10.5\right)$ and $P a G l$ by pain $(0.80,<0.0001)$ and fatigue $(0.17,<0.001)\left(R^{2}=0.89, p<0.001, S E E=12.4\right)$. Swollen and tender joint count, CRP and PhGl did not add to the explanation of the patient-reported VAS scores. The bias [LLoA; ULoA] for FTG versus pain was $8.5 \pm 19.1(p<0.0001)$ [-29.1; 45.9], for FTG versus PaGl 4.1 $\pm 19.4(p<0.05)[-34.0 ; 42.2]$ and for PaGl versus pain $4.4 \pm 11.5(p<0.0001)[-18.1 ; 26.9]$. Thus biases were small but limits of agreement were pronounced.

Conclusions: In patients with PsA, VAS FTG, pain and PaGl were nearly identical and were strongly inter-associated on the group level with no explanatory influence of more objective measures. However, on the individual patient level substantial discrepancies between the VAS scores were observed. The findings emphasise the complexity of understanding and dealing with PROMs in the daily clinic.

Disclosure of Interest: None declared

DOI: 10.1136/annrheumdis-2018-eular.3546 